\title{
0556 TRANSPORT-RELATED WALKING AND PEDESTRIAN INJURIES AMONG ADULTS IN URBAN INDIA: ASSOCIATION WITH SOCIOECONOMIC INDICATORS
}

R Dandona*, G A Kumar, L Dandona Correspondence: Public Health Foundation of India, PHD House, 4/2, Sirifort Institutional Area August Kranti Marg, New Delhi 110016, India

\subsection{6/ip.2010.029215.556}

Objective The association of individual- and cluster-level socio-economic status indicators for transport-related walking (TRW) and pedestrian injuries is reported from an urban population in India.

Methods A three-stage systematic cluster design was used to recruit 7650 people aged $15-49$ years (92.8\% participation) representative of Hyderabad city population. Participants responded to questions on TRW and pedestrian injuries. Logistic regression was used to assess association for having $>2$ TRW trips per day and non-fatal pedestrian injury incidence in the last 12 months with individual-level (caste, education, per capita monthly income (PCMI), household ownership of vehicle) and cluster-level (cluster median income, CMI) socioeconomic indicators.

Results The overall mean number of TRW trips per day was 6.25 with five times higher trips in the households that owned only a cycle as compared with those which owned a car $(p<0.001)$. Those with no education had the highest odds of reporting $>2$ TRW trips per day (8.30; 95\% CI 6.79 to 10.13), and these trips decreased with increasing PCMI and CMI quartiles. Annual age-sex-adjusted rate for non-fatal pedestrian injuries was $7.4 \%$ (95\% CI 6.7 to 8.0 ) and the highest rate was reported for those with $>10$ TRW trips per day $(15.1 \%$, 95\% CI 12.4 to 17.8). The odds of reporting non-fatal pedestrian 


\section{IP Safety 2010 abstracts}

injury in the last 12 months increased with increasing number of TRW trips.

Conclusions These data can contribute to understanding the differences in walking patterns and pedestrian injuries across the socio-economic gradient to formulate police and interventions aimed at reducing road traffic injuries in India. 